University of Nebraska - Lincoln

DigitalCommons@University of Nebraska - Lincoln

\title{
Multi-platform comparison of ten commercial master mixes for probe-based real-time polymerase chain reaction detection of bioterrorism threat agents for surge preparedness
}

\author{
Gregory S. Buzard \\ National Center for Emerging and Zoonotic Infectious Diseases (NCEZID) \\ Daniel Baker \\ National Center for Emerging and Zoonotic Infectious Diseases (NCEZID) \\ Mark J. Wolcott \\ United States Army Medical Research Institute for Infectious Diseases \\ David A. Norwood \\ United States Army Medical Research Institute for Infectious Diseases \\ Leslie A. Dauphin \\ National Center for Emerging and Zoonotic Infectious Diseases (NCEZID), Ldauphin@CDC.GOV
}

Follow this and additional works at: https://digitalcommons.unl.edu/usarmyresearch

Buzard, Gregory S.; Baker, Daniel; Wolcott, Mark J.; Norwood, David A.; and Dauphin, Leslie A., "Multiplatform comparison of ten commercial master mixes for probe-based real-time polymerase chain reaction detection of bioterrorism threat agents for surge preparedness" (2012). US Army Research. 188. https://digitalcommons.unl.edu/usarmyresearch/188

This Article is brought to you for free and open access by the U.S. Department of Defense at DigitalCommons@University of Nebraska - Lincoln. It has been accepted for inclusion in US Army Research by an authorized administrator of DigitalCommons@University of Nebraska - Lincoln. 


\title{
Multi-platform comparison of ten commercial master mixes for probe-based real-time polymerase chain reaction detection of bioterrorism threat agents for surge preparedness
}

\author{
Gregory S. Buzard ${ }^{\mathrm{a}, \mathrm{c}}$, Daniel Baker ${ }^{\mathrm{b}}$, Mark J. Wolcott ${ }^{\mathrm{c}}$, David A. Norwood ${ }^{\mathrm{c}}$, Leslie A. Dauphin ${ }^{\mathrm{a}, *}$

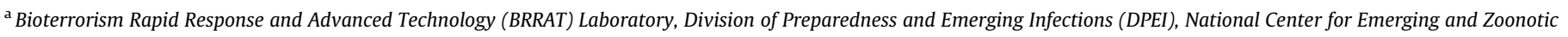 \\ Infectious Diseases (NCEZID), Centers for Disease Control and Prevention (CDC),1600 Clifton Road, Atlanta, GA 30333, United States

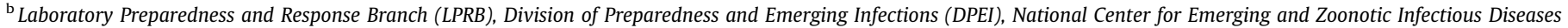 \\ (NCEZID), Centers for Disease Control and Prevention (CDC),1600 Clifton Road, Atlanta, GA 30333, United States \\ ${ }^{\mathrm{c}}$ Diagnostic Systems Division (DSD), United States Army Medical Research Institute for Infectious Diseases (USAMRIID), Porter Street, Ft. Detrick, MD 21702, United States
}

\section{A R T I C L E I N F O}

\section{Article history:}

Received 5 April 2012

Received in revised form 14 August 2012

Accepted 3 October 2012

Available online 26 October 2012

\section{Keywords:}

Master mix

Real-time PCR

Bioterrorism

Surge preparedness

Select agents

\begin{abstract}
A B S T R A C T
The Centers for Disease Control and Prevention and United States Army Research Institute for Infectious Diseases have developed real-time PCR assays for the detection of bioterrorism threat agents. These assays all rely on a limited number of approved real-time PCR master mixes. Because the availability of these reagents is a critical element of bioterrorism preparedness, we undertook a joint national preparedness exercise to address the potential surge needs resulting from a large-scale bio-emergency. We identified 9 commercially-available potential alternatives to an existing approved master mix (LightCycler FastStart DNA Master HybProbes): the TaqMan Fast Universal PCR master mix, OmniMix HS, FAST qPCR master mix, EXPRESS qPCR SuperMix kit, QuantiFast Probe PCR kit, LightCycler FastStart DNA Master ${ }^{\text {PLUS }}$ HybProbe, Brilliant II FAST qPCR master mix, ABsolute Fast QPCR Mix and the HotStart IT Taq master mix. The performances of these kits were evaluated by the use of real-time PCR assays for four bioterrorism threat agents: Bacillus anthracis, Brucella melitensis, Burkholderia mallei and Francisella tularensis. The master mixes were compared for target-specific detection levels, as well as consistency of results among three different real-time PCR platforms (LightCycler, SmartCycler and 7500 Fast Dx). Realtime PCR analysis revealed that all ten kits performed well for agent detection on the 7500 Fast Dx instrument; however, the QuantiFast Probe PCR kit yielded the most consistently positive results across multiple real-time PCR platforms. We report that certain combinations of commonly used master mixes and instruments are not as reliable as others at detecting low concentrations of target DNA. Furthermore, our study provides laboratories the option to select from the commercial kits we evaluated to suit their preparedness needs.
\end{abstract}

Published by Elsevier Ireland Ltd.

\section{Introduction}

The advent of real-time quantitative PCR has transformed the discipline of pathogenic diagnostics. In comparison to conventional PCR, real-time PCR assays offer a greater detection range and reproducibility, with significantly less inter-assay variation [1-4]. With its popularity, there has been rapid growth of commerciallyavailable real-time PCR master mixes and detection platforms, which greatly increase the options available to laboratories [5,6]. These advances in options and technology have generated a need

\footnotetext{
* Corresponding author at: BRRAT Laboratory, LPRB, DPEI, NCEZID, CDC, Mail Stop G-42, 1600 Clifton Road, Atlanta, GA 30333, USA. Tel.: +1 404639 4991; fax: +1 4046394234 .

E-mail address: Ldauphin@CDC.GOV (L.A. Dauphin).
}

for a periodic comparative evaluation, and such an opportunity recently arose as part of a Joint National Preparedness Exercise conducted by laboratories of the United States Army Research Institute for Infectious Diseases (USAMRIID) and the Centers for Disease Control and Prevention (CDC).

The Bioterrorism Rapid Response and Advanced Technology (BRRAT) Laboratory of the CDC is charged with improving our capabilities to detect Biological Select Agents and Toxins (BSAT) that could be used as weapons of mass terror and warfare [7]. The BRRAT laboratory, in collaboration with selected CDC subject matter experts, has developed a number of real-time PCR assays for BSAT detection [8]. These assays are deployed to reference laboratories of the Laboratory Response Network (LRN), a network of more than 160 state, federal and local public health, Department of Defense (DOD), and veterinary laboratories that are poised nationwide to rapidly detect and identify potential bio-threats [8]. 
The LRN performs detection and identification assays for several category A and B BSAT, including but not limited to Bacillus anthracis, Yersinia pestis, Francisella tularensis, Burkholderia spp. and Brucella spp. USAMRIID has independently developed assays for these BSAT for use by the Department of Defense.

This joint study came about because the availability and efficacy of our detection reagents are critical elements of our bioterrorism preparedness [7]. During a catastrophic bioterrorism attack or a naturally occurring pandemic, such reagents might become limiting. As a matter of interagency planning for such potential large-scale events, CDC and USAMRIID collaborated to identify alternative master mixes that could, in the face of an emergency, supplement our existing supplies of critical real-time PCR reagents to insure that there would be sufficient deliverable reagents to address any additional needs.

The goal of this study was to compare the performance of 9 newer commercial real-time PCR master mixes to our current approved master mix for their ability to amplify and detect various sizes and kinds of PCR targets from four representative BSAT organisms: $B$. anthracis, $B$. melitensis, $B$. mallei and $F$. tularensis. The kits were evaluated, with their corresponding real-time PCR assays, by direct comparisons of the average cross-threshold $\left(C_{T}\right)$ values under the demanding condition of DNA concentrations approaching the limit of detection (LOD) for each BSAT. In addition, the master mixes were evaluated for their cross-platform portability by the use of three real-time PCR platforms.

\section{Materials and methods}

\subsection{Biosafety procedures}

All live specimen manipulations for DNA preparations were performed at USAMRIID or the Critical Reagents Program (CRP) within biosafety level 3 (BSL-3) facilities, as required, using practices outlined in the "Biosafety in Microbiological and Biomedical Laboratories 5th Edition" [9]. PCR assays were conducted in BSL-2 facilities.

\subsection{Commercial master mix kits}

A market analysis was performed to identify commercial vendors that could supply new master mix kits based on predetermined commercial capacity and composition criteria. The goal was to identify suitable alternatives to our standard real-time PCR master mix, the LightCycler FastStart DNA Master HybProbe kit (Roche Applied Science, Indianapolis, IN), for emergency use in a surge event. Possible vendors were identified that possessed viable candidates. A set of modifiable scoring criteria was created based on the projected needs of the LRN. The criteria for determining suitable "surge supplements" was that the new reagent(s) should be able to substitute for existing LRN and USAMRIID validated reagents in all of their Standard Operating Protocols (SOP) for BSAT detection, with a minimum of changes to their SOP (i.e., they should be able to fulfill a protocol plug-in-and-play scenario), fulfilling the same role as the current master mix without changes to cycling parameters that would necessitate a revalidation of approved protocols or risk procedural differences leading to unintentional ambiguity. A total of 9 such master mixes were identified for comparative evaluation (Table 1 )

The LightCycler FastStart DNA Master Hybridization Probes $(10 \times)$ kit (Roche), henceforth referred to as Roche I, was provided with LightCycler - FastStart enzyme (vial 1a) and LightCycler-FastStart Reaction Mix (vial 1b), which were first combined as directed, then mixed with appropriate amounts of $25 \mathrm{mM} \mathrm{MgCl} 2$ (usually to $4 \mathrm{mM}$ ). The LightCycler FastStart DNA Master Hybridization Probes ${ }^{\text {PLUS }}$ $5 \times$ kit (Roche II) comes with the $\mathrm{MgCl}_{2}$ added to the buffer, which is complete after adding the $5 \times$ polymerase. The other 8 master mixes come as a $2 \times$ concentration with the DNA polymerase, pre-optimized $\mathrm{MgCl}_{2}$, and reaction buffer pre-combined in a single tube.

\subsection{Culture and DNA extraction of BSATs}

The Unified Culture Collection (UCC) is a library of BSAT organisms and their nucleic acid extracts that is maintained by the DSD of USAMRIID for the Critical Reagents Program (CRP) of the Aberdeen Proving Ground-Edgewood Area, MD. DNA of four representative category A and B BSAT (provided from the CRP by Gerald Howe of DSD-USAMRIID) were used in this study: B. anthracis strain Ames (UCC, DNA-BACI008), B. melitensis strain 16M (UCC, BRUC013D-CRP07), B. mallei strain 010 (UCC, BURK010D-CRP07) and F. tularensis strain LVS (UCC, DNA-FRAN004). Genome sizes used to determine LODs for each BSAT were obtained from GenBank (http://www.ncbi.nlm.nih.gov/sites/entrez) and as described [10-12].

The DNA was extracted directly from bacterial cultures and was then safetytested against the presence of viable organisms before use. These DNA were cesium chloride gradient purified, quantified, and dispensed as $5 \mathrm{ng}$ of DNA per vial, then stored at $-70{ }^{\circ} \mathrm{C}$. We performed the appropriate calculations to determine the genome copy (gc) equivalents per nanogram of DNA for each organism and resuspended the DNA accordingly to $100,000 \mathrm{gc} / \mu \mathrm{l}$ in $10 \mathrm{mM}$ Tris (pH 8.0). Ten-fold serial dilutions of the DNA were then prepared to obtain four log-order differences of concentration of target DNA for each organism (i.e., 20,000, 2000, 200 and 20 gc per reaction). Further dilutions in Tris were made as needed.

\subsection{Real-time PCR}

The real-time PCR assays used in this study have been used by USAMRIID for the in vitro qualitative presumptive detection of BSAT DNA in environmental samples, clinical specimens and culture isolates and have been described in previous reports [13-15]. We used the following four primer and probe sets: BALB (chrom.), which detects a chromosomal gene of B. anthracis; omp2a, targeting the outer membrane protein $2 a$ gene of $B$. melitensis; bimAma, targeting an actin polymerization gene of $B$. mallei; and tul4, for $F$. tularensis (Table 2). Prior to rehydration, dry primers and probes were stored at $2-8{ }^{\circ} \mathrm{C}$ in the dark. Each tube was rehydrated to appropriate concentrations with $10 \mathrm{mM}$ Tris ( $\mathrm{pH}$ 8.0). The rehydrated aliquots of primers and light-sensitive probes were stored in the dark at $2-8{ }^{\circ} \mathrm{C}$ for the duration of the study. The four signature sets were tested against a 10 -fold dilution series, as described above, of their purified target DNAs.

Real-time PCR was conducted according to the agent-specific SOPs. The final volume of each reaction mixture was $25 \mu \mathrm{l}$ and was comprised of an average of $4 \mathrm{mM} \mathrm{MgCl}_{2}$ (range 3-8 mM), 200-1000 nM specific primers (as SOP specified), 25$200 \mathrm{nM}$ TaqMan probe, with $5 \mu \mathrm{l}$ of purified BSAT template DNA to give the desired gc number per reaction volume, and $5 \mu \mathrm{l}$ of PCR-grade water or Tris to the notemplate-control reactions. Thermal cycling was performed using the following program: one cycle at $95{ }^{\circ} \mathrm{C}$ for $8 \mathrm{~min}$ for Hot-Start Taq activation, followed by 45 cycles of $95^{\circ} \mathrm{C}$ for $5 \mathrm{~s}$ and $60{ }^{\circ} \mathrm{C}$ for $30 \mathrm{~s}$. A fluorescence reading was taken at the end of each $60{ }^{\circ} \mathrm{C}$ step. These same cycle conditions were used for all instruments used in this study.

Initial rounds of testing were conducted using the 7500 Fast Dx instrument (Applied Biosystems, Foster City, CA) with the Roche I master mix, to obtain a relative baseline LOD for each BSAT. The average $C_{T}$ values at each DNA concentration were calculated from triplicate samples at each DNA concentration. The LOD was determined to be the lowest concentration for which triplicate samples resulted in a similar positive result, as measured by $C_{T}$ values $\leq 45$. Further performance testing of the 10 master mixes was done, conducted as described above with the 7500 Fast Dx, but at a single DNA concentration that was three times the experimentally established LOD $(3 \times$ LOD). The kits were then ranked for performance based on comparisons of mean $C_{T}$ values.

Table 1

Summary of the ten commercial master mix kits evaluated in this study.

\begin{tabular}{|c|c|c|}
\hline Manufacturer & Master mix kit & Catalog number \\
\hline Applied Biosystems (ABI) & TaqMan Fast Universal PCR master mix & 4352042 \\
\hline Cepheid & OmniMix HS & OMNL1-100N-050 \\
\hline Eurogentec & FAST qPCR master mix plus Low ROX & RT-QP2X-03 + WOULRF-2 \\
\hline Invitrogen & EXPRESS qPCR SuperMix kit & $11785-200$ \\
\hline Qiagen & QuantiFast Probe PCR + ROX kit & 204352 \\
\hline Roche (I) & LightCycler FastStart DNA Master HybProbe & $12-239-272-001$ \\
\hline Roche (II) & LightCycler FastStart DNA Master ${ }^{\text {PLUS }}$ HybProbe & 3515575001 \\
\hline Stratagene & Brilliant II FAST QPCR master mix with Low ROX & 600806 \\
\hline Thermo Fisher Scientific & ABsolute Fast QPCR Mix Plus ROX & $A B-4325-A$ \\
\hline USB & HotStart-IT Taq master mix & 71196 \\
\hline
\end{tabular}


Table 2

Primer and probe sequences for real-time PCR assays used to compare ten commercial master mixes for detection of bioterrorism threat agents.

\begin{tabular}{|c|c|c|c|}
\hline Primer or probe ${ }^{a}$ & Target & Nucleotide sequence $\left(5^{\prime} \rightarrow 3^{\prime}\right)$ & Amplicon size (bp) \\
\hline BALB-F41 & \multirow[t]{3}{*}{ B. anthracis (chromosome) } & TGGCGGAAAAGCTAATATAGTAAAGTA & \multirow[t]{3}{*}{106} \\
\hline BALB-R-146 & & CСACATATCGAATCTCCTGTCTAAAA & \\
\hline BALB-P-88-MGB & & ACTTCTAAAAAGCAGATAGAAAT & \\
\hline OMP2a-F1755 & \multirow[t]{3}{*}{ B. melitensis (outer membrane protein $2 \mathrm{a}$ ) } & CCAGGCGTACCGGTTATCTC & \multirow[t]{3}{*}{101} \\
\hline OMP2a-R1845 & & AGACCCTTTTGAGGTCTACTCCCTTA & \\
\hline OPM2A-p1799-MGB & & TGGTCGAAGGCGCTC & \\
\hline BMSEC3-F-49 & \multirow[t]{3}{*}{ B. mallei (polymerase gene) } & CAGTTGATTCTCCCACC & \multirow[t]{3}{*}{96} \\
\hline BMSEC3-R-144 & & TGTCTTGTTGAGCATGAGA & \\
\hline BMSEC3-P-104R-MGB & & CATACGGATGTATAGAACCAAT & \\
\hline Tul4-F-774 & \multirow[t]{3}{*}{ F. tularensis (tul4 gene) } & CAGCATACAATAATAACCCACAAGG & \multirow[t]{3}{*}{103} \\
\hline Tul4-R-876 & & TCAGCATACTTAGTAATTGGGAAGC & \\
\hline Tul4-P-809S & & TTACAATGGCAGGCTCCAGAAGGTT & \\
\hline
\end{tabular}

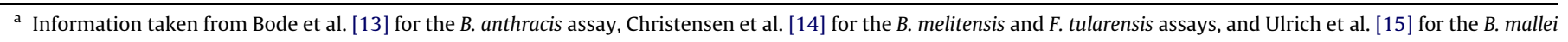
assay.

\subsection{Comparison of the master mixes on two additional real-time PCR platforms}

Based on the above testing results, surge candidates were next evaluated for performance on two additional platforms, the SmartCycler (Cepheid, Sunnyvale, CA) and the LightCycler (Roche). Performance testing was again conducted in triplicate for each signature group at $3 \times$ LOD. For each BSAT, the mean $C_{T}$ values for triplicate wells were calculated for each master mix, and these values were compared to each other and to those of the approved Roche I master mix.

\subsection{Data analysis}

The real-time PCR data was analyzed using the appropriate software supplied for each platform. For example, the 7500 Fast System Dx SDS with v1.4 21 CFR Part 11 Module software (Applied Biosystems) using the 'Delta Rn versus Cycle' mode. For the LightCycler, each capillary tube was read in Channel 1 (F1) at a gain setting of 16 . The data was analyzed with LightCycler data analysis software version 3.5.3 (Roche). Sample curves were analyzed by using the second derivative maximum with the baseline adjustment set to arithmetic. For the SmartCycler, data were analyzed using the SmartCycler software version 1.2d (Cepheid). The SmartCycler settings consisted of a primary curve analysis with a manual threshold setting of 10 , background subtraction turned on, boxcar average set to five cycles, background minimum cycle set to 5 , and background maximum cycle set to 45 . Real-time PCR results with $C_{T}$ values greater than 45 were considered negative.

\section{Results}

\subsection{Real-time PCR limit of detection for four BSAT}

The LOD for the four BSAT was determined with the Roche I master mix as a baseline for comparative evaluations of the 9 master mixes identified in this study. Fig. 1 shows the real-time PCR LOD for B. anthracis, B. melitensis, B. mallei and F. tularensis on the 7500 Fast Dx instrument. Generally, the LODs for the four BSATs were comparable with the Roche I master mix. The LOD for B. anthracis, B. melitensis, and B. mallei was $10 \mathrm{gc}$, while the LOD for F. tularensis was $5 \mathrm{gc}$.

\subsection{Comparison of the ten master mixes on the 7500 Fast Dx instrument}

Table 3 shows real-time PCR results for the 10 commercial master mixes on the 7500 Fast Dx instrument, with $C_{T}$ values corresponding to three times the experimentally determined LOD for the four BSATs. Taken as a whole, most of the 9 master mix candidates were roughly equivalent or superior to the currently used Roche kit (Roche I) with each BSAT. However, there were four kits that ranked in the top three for performance on the 7500 Fast Dx with two or more agents: the ABI, Eurogentec, Invitrogen, and Qiagen kits.

Individually, for the $B$. anthracis detection assay, there were 6 master mixes that performed as well as or better than the Roche I master mix. The Invitrogen kit ranked first, with the lowest average $C_{T}$ value, followed by the $\mathrm{ABI}$ and Eurogentec master mixes, whereas the Stratagene kit ranked last with the highest average $C_{T}$ value. A total of 8 master mixes performed as well or better than the Roche I master mix for $B$. melitensis, with two pairs that resulted in equivalent results (ABI with Thermo Fisher Scientific and Cepheid with USB). The top three master mixes for $B$. mallei detection were ABI, Eurogentec, and Thermo Fisher Scientific, whereas the Cepheid master mix resulted in the worst performance. As for F. tularensis, five master mixes performed as well or better than the Roche I master mix, with the Qiagen master mix ranking first and three master mixes tied for last place (Cepheid, Roche II and Stratagene). There was no significant difference in mean $C_{T}$ values between the Roche I master mix and the 9 other master mixes for B. anthracis, B. mallei and F. tularensis; however, the Qiagen master mix resulted in significantly better detection for $B$. melitensis than the Roche I kit [as measured by $\geq 1$ log-unit difference in detection levels $\left(C_{T}\right.$ value difference $\left.\geq 3.3\right)$ ].

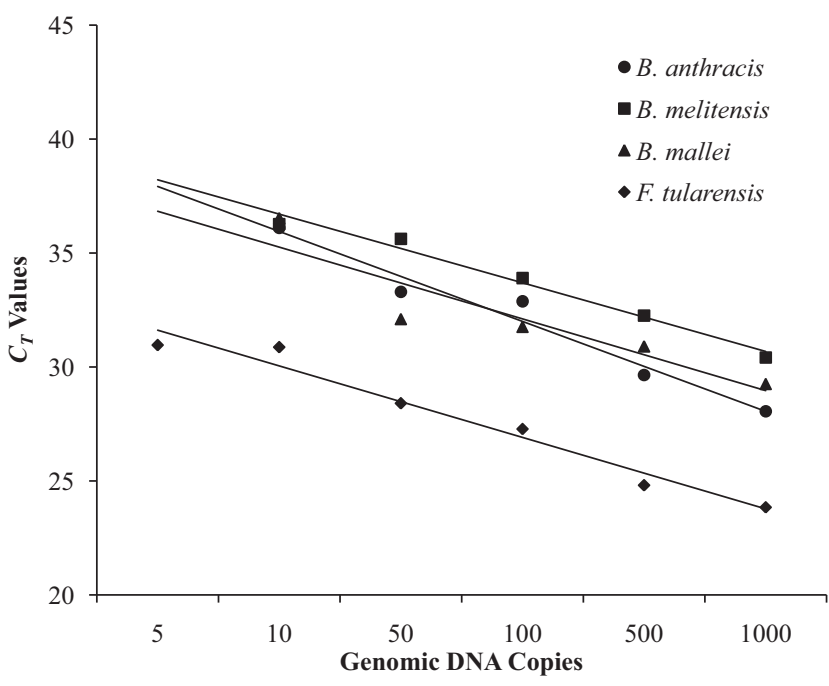

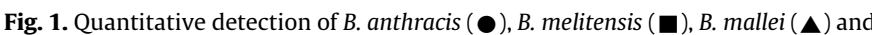
F. tularensis $(\bullet)$, using the real-time PCR assays described by Bode et al. [13], Christensen et al. [14] and Ulrich et al. [15] with the LightCycler FastStart DNA Master HybProbe (Roche I). Serial dilutions of purified bacterial DNA from each bioterrorism threat agent were prepared, and the assays were performed in triplicate with samples at each concentration on the 7500 Fast Dx instrument. The data was plotted as the mean of $C_{T}$ values for triplicate samples versus the genomic DNA copy numbers. The limit of detection (LOD) was determined to be the lowest concentration for which three out of three replicates produced a positive result. 
Table 3

Comparison of ten commercial master mixes for detection of bioterrorism threat agents by real-time PCR.

\begin{tabular}{|c|c|c|c|c|c|c|c|c|}
\hline \multirow[t]{2}{*}{ Master mix } & \multicolumn{8}{|c|}{ Average $C_{T}(\text { mean } \pm \mathrm{SD})^{\mathrm{a}}$} \\
\hline & B. anthracis & Ranking & B. melitensis & Ranking & B. mallei & Ranking & F. tularensis & Ranking \\
\hline $\mathrm{ABI}$ & $33.9 \pm 0.46$ & 2 & $30.2 \pm 0.15$ & 5 & $30.0 \pm 0.16$ & 1 & $28.1 \pm 0.14$ & 4 \\
\hline Cepheid & $35.4 \pm 0.84$ & 8 & $30.4 \pm 0.12$ & 6 & $33.7 \pm 0.80$ & 10 & $29.2 \pm 0.37$ & 8 \\
\hline Eurogentec & $34.0 \pm 0.50$ & 3 & $29.2 \pm 0.34$ & 3 & $30.2 \pm 0.08$ & 2 & $27.7 \pm 0.15$ & 3 \\
\hline Invitrogen & $33.5 \pm 1.59$ & 1 & $29.5 \pm 0.43$ & 4 & $31.2 \pm 0.61$ & 4 & $27.5 \pm 0.41$ & 2 \\
\hline Qiagen & $34.5 \pm 0.27$ & 6 & $27.1 \pm 0.81$ & 1 & $31.5 \pm 2.63$ & 5 & $27.2 \pm 0.41$ & 1 \\
\hline Roche I & $35.3 \pm 0.53$ & 7 & $31.9 \pm 0.56$ & 7 & $32.9 \pm 0.40$ & 8 & $28.4 \pm 0.31$ & 6 \\
\hline Roche II & $36.5 \pm 0.37$ & 9 & $32.6 \pm 0.34$ & 8 & $33.3 \pm 0.46$ & 9 & $29.2 \pm 0.33$ & 8 \\
\hline Stratagene & $36.8 \pm 0.64$ & 10 & $29.0 \pm 0.19$ & 2 & $31.8 \pm 0.35$ & 6 & $29.2 \pm 0.41$ & 8 \\
\hline Thermo FS & $34.5 \pm 1.13$ & 5 & $30.2 \pm 0.33$ & 5 & $30.6 \pm 0.70$ & 3 & $28.2 \pm 0.28$ & 5 \\
\hline USB & $34.3 \pm 0.21$ & 4 & $30.4 \pm 0.08$ & 6 & $32.0 \pm 0.61$ & 7 & $28.6 \pm 0.28$ & 7 \\
\hline
\end{tabular}

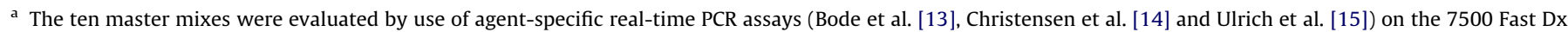

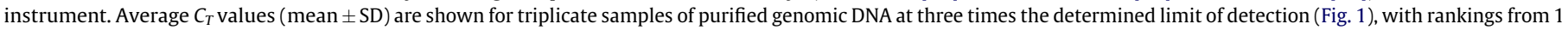
to 10 in order of increasing $C_{T}$ values.

\subsection{Evaluation of the ten master mixes on multiple real-time PCR platforms}

The 10 master mixes were further evaluated for BSAT detection on two additional real-time PCR instruments. Fig. 2 shows the realtime PCR results for $B$. anthracis, $B$. melitensis, $B$. mallei and $F$. tularensis at $3 \times$ LOD with the 7500 Fast Dx, LightCycler and SmartCycler platforms. By and large there was great variability in BSAT detection among the three instruments, and several master mix failures for detection on the LightCycler platform. For $B$. anthracis, all 10 of the master mixes resulted in positive results on the 7500 Fast Dx and the SmartCycler instruments; however, four of the master mixes, the $\mathrm{ABI}$, Eurogentec, Invitrogen, and Stratagene, resulted in negative results on the LightCycler (Fig. 2A). Similarly, all of the master mixes demonstrated crossplatform portability between the 7500 Fast Dx and SmartCycler platforms, but three failed to detect $B$. melitensis on the LightCycler (ABI, Eurogentec, and Stratagene) (Fig. 2B). A total of 5 master mixes failed for detection of $B$. mallei on the LightCycler instrument, while all 10 resulted in positive results on the 7500 Fast Dx and SmartCycler (Fig. 2C). Likewise, all 10 master mixes resulted in positive results for $F$. tularensis on the 7500 Fast Dx and SmartCycler instruments, whereas three failed on the LightCycler (ABI, Eurogentec and Stratagene) (Fig. 2D).

\subsection{Comparison of costs and pipetting steps}

Table 4 shows comparison of costs and number of pipetting steps between the commercial master mix kits. The kits were ranked from 1 to 10 in order of increasing cost per PCR reaction. Of the 10 master mix kits, the USB kit was the least expensive, followed by the Eurogentec kit, then the ABI kit. The Cepheid

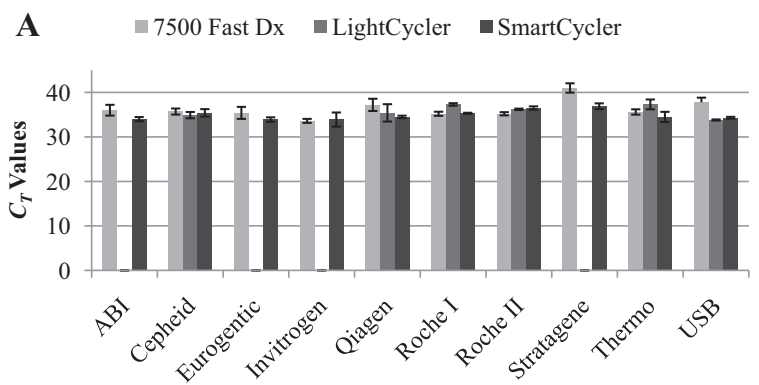

Master Mix Kit

\section{B}

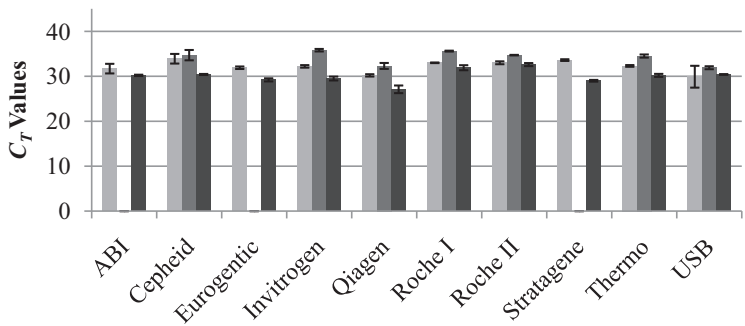

Master Mix Kit
C $\square 7500$ Fast Dx $\square$ LightCycler $\square$ SmartCycler

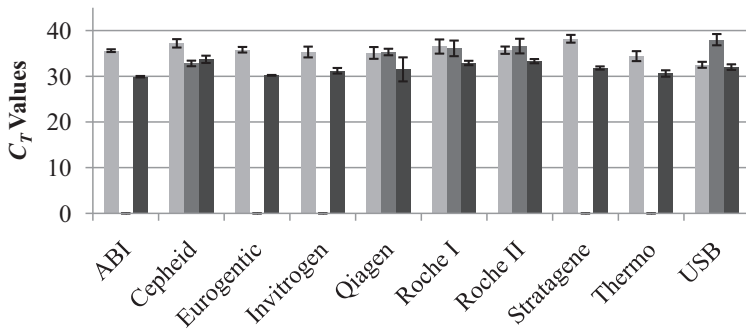

Master Mix Kit

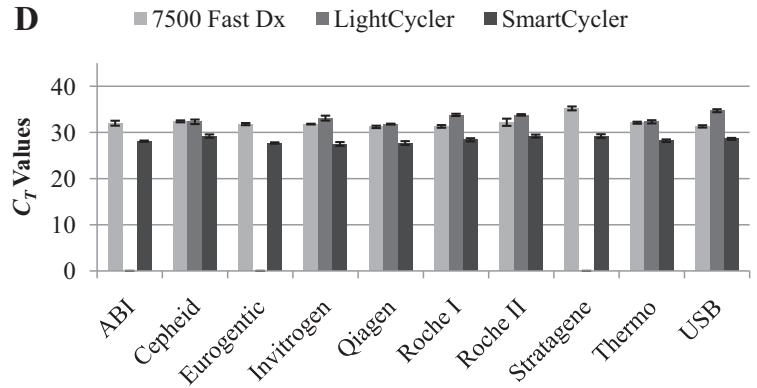

Master Mix Kit

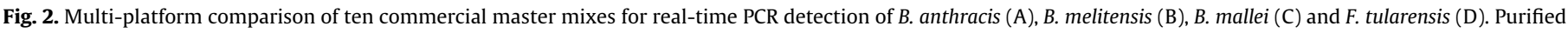

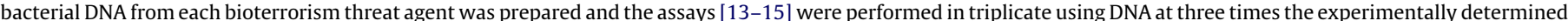

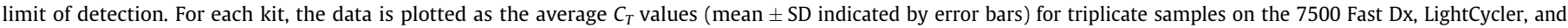
SmartCycler instruments. 
Table 4

Comparison of costs and pipetting steps between the ten commercial master mix kits.

\begin{tabular}{lcl}
\hline Manufacturer & Ranking for Cost/PCR reaction ${ }^{\mathrm{a}}$ & No. of pipetting steps $^{\mathrm{c}}$ \\
\hline ABI & 2 & 1 \\
Cepheid & 10 & 1 \\
Eurogentec & 3 & 1 \\
Invitrogen & 6 & 1 \\
Qiagen & 9 & 1 \\
Roche I & $8^{\mathrm{b}}$ & 2 \\
Roche II & $7^{\mathrm{b}}$ & 3 \\
Stratagene & 4 & 1 \\
Thermo FS & 5 & 1 \\
USB & 1 & 1 \\
\hline
\end{tabular}

a The cost per reaction was calculated by dividing the cost for each commercial kit, based on the manufacturer's list price in summer of 2011 (U.S. dollars), by the number of PCR reactions ( $25 \mu \mathrm{l}$ each) that could be performed with each kit. The kits were ranked from 1 to 10 based on cost per PCR reaction, with 1 corresponding to the least expensive and 10 for the most expensive.

b These two mixes were the same price per reaction.

c Pipetting steps include those unique to a master mix, such as mixing from separate tubes of polymerase, magnesium chloride and reaction buffer, where applicable.

master mix kit was by far the most expensive per assay of the kits evaluated in this study.

The master mix kits were also compared for the number of pipetting steps required to set up a PCR reaction. This comparison only included steps that were unique to a master mix and excluded all common steps (e.g., addition of template DNA). With the exception of the two Roche master mix kits, all of the kits were provided as one-step PCR reaction mixtures; the Roche I kit required three pipetting steps, while the Roche II kit required two.

\section{Discussion}

The LRN currently has only two master mix kits validated to be used for their BSAT testing; by far the primary one is the LightCycler FastStart DNA Master HybProbes (Roche), but in one unusual agent-specific case the Fast Universal PCR master mix (Applied Biosystems) can be used as well. USAMRIID is faced with a similar challenge, with its primary use of a Platinum Taq mixture. A potential concern arose that there might not be enough deliverable master mix reactions in stock, either in surge capacity storage by the CDC or at the manufacturer. As part of our ongoing efforts to improve surge preparedness, we sought to identify additional realtime PCR master mixes that could be used in the case of a largescale emergency event. Therefore, in this joint agency effort between CDC and USAMRIID, we compared 9 new kits to our current kit as candidates for such an emergency use.

Previous studies have compared either the performance characteristics of a small number of commercially-available real-time PCR reagents or compared only a small number of signature sets. In 1998, Al-Soud and Radstrom [16] evaluated 9 different PCR enzymes and their associated reaction buffers (the precursors to today's master mixes). Their study [16] used one signature set and examined the ability of certain PCR enzymes to amplify in the presence of common PCR-inhibiting components found in complex biological samples. Subsequently, Wolffs et al. [17] investigated four DNA polymerases (Taq, DyNazyme, Tth and rTth) and two different buffer systems in real-time PCR and showed that the choice of both the type DNA polymerase and the buffer system affected amplification efficiency. In our study, we compared 10 commercial reagents by the use of real-time PCR assays for four different select agents in an effort to provide a thorough evaluation of the most popular kits that are currently on the market. Real-time PCR $C_{T}$ values were used for quantitative comparisons of master mix reagent performance; however, it should be noted that quantitative detection is not necessary for routine diagnostics or during a bio-threat emergency when only the presence or absence of the target agent is in question.

Successful detection and identification of BSAT organisms depends crucially on both the quantity and quality of the DNA samples being analyzed [5]. In this study, highly purified DNA templates were used to determine the LOD for the four BSATs on the 7500 Fast Dx instrument. Although it is unlikely that highly purified DNA would be used as template during a bio-threat emergency, we chose this approach because it offers several advantages for master mix reagent evaluations, including the ability to accurately convert DNA concentration to genomic copy numbers, and it reduces the potential bias introduced by inhibitory contaminants left by some DNA extraction processes. Our results showed that, with these conditions, most of the new master mix kits performed as well or better on the 7500 Fast Dx than the current Roche master mix for all four BSATs. However, the individual kit results were not always uniformly equivalent, in that several kits ranked among the top three for one agent and its signature, but not for others (Table 3 ). These results indicate that even when the DNA is highly purified, as in the case of our experiments, the choice of and concentration of reaction mix components, such as primers, probes, analytical platforms, and cycling conditions, can affect assay sensitivity. For the purposes of this study, a significant difference in master mix reagent performance was defined as $C_{T}$ value differences $\geq 3.3$. Although this criterion was acceptable for evaluating and ranking differences in detection levels, is important to note that it is not acceptable for all applications, such as studies involving gene expression where smaller differences between cycle numbers could be relevant.

More relevant to our current study, Sohni et al. [5] recently evaluated 5 commercial TaqMan PCR reagent kits in assays for $B$. anthracis. Their study showed that three of the 5 kits performed better for B. anthracis detection. However, a limitation of their study was that it was done on a single type of real-time PCR platform. Previous studies have reported significant variability in PCR efficiency and results among different types of thermal cyclers $[18,19]$. There are three different types of LRN-approved real-timePCR detection platforms, represented in this study by the LightCycler (Roche), the SmartCycler (Cepheid) and the 7500 Fast Dx (Applied Biosystems). We chose to do our evaluations on these instruments after careful thought regarding the varying detection throughput capacities in diagnostic laboratories; the SmartCycler is a 16-reaction vessel per block instrument, the LightCycler has a 32-sample capacity, and the 7500 Fast Dx has a 96-well capacity.

Most PCR systems employ pre-determined settings and proprietary master mixes that differ from one system to another. These differences affect detection, as Lu et al. [20] showed, comparing the 7500 (Applied Biosystems) and the LightCycler 480 (Roche) instruments, using their default settings, proprietary reagents, ramp rates and magnesium concentrations. Two targets readily detected by the 7500 system were not detected using the LightCycler 480 system [20]. Our findings were consistent with those observations, as three of the kits that performed best in our initial studies on the 7500 Fast Dx, the ABI, Eurogentec and Invitrogen kits, failed on the LightCycler (Table 3 and Fig. 2).

We hypothesize that these failures on the LightCycler are attributed to the absence of bovine serum albumin in these master mixes, which is required to prevent absorption of the Taq polymerase to the glass capillaries used with the LightCycler instrument. Additionally, Bentley et al. [21] demonstrated that several critical parameters might need to be optimized when converting between real-time PCR platforms, including optimization of the $\mathrm{MgCl}_{2}$ concentration and changes in PCR cycling parameters, such as dwell times and ramping speeds. This is not always the case, however. Christensen et al. [14] compared the 
performance of assays for 7 biological threat agents on the R.A.P.I.D. (Idaho Technology, Inc., Salt Lake City, UT) the LightCycler, and the SmartCycler, and found that their assays gave comparable results for performance, sensitivity and specificity on all three platforms. Based on the results of this study [14], and to meet our selection criteria of maintaining current BSAT assay conditions, we evaluated the master mixes on three real-time PCR instruments using identical thermal cycling conditions.

The primary real-time PCR master mix that is currently used for the LRN's BSAT assays comes in three tubes that must be mixed together prior to setting up the rest of the PCR reactions. In 8 of the newer commercial master mix systems, these three components are pre-mixed into one tube (Table 4 ). In the 9 new candidate kits we compared, the concentration of magnesium was already optimized for the master mix and no additional adjustments were needed or made. The concentrations of polymerase, nucleotides, magnesium and buffers in these newer master mixes are also pre-optimized for the faster PCR ramping conditions now widely utilized. Pre-combining them in a single tube reduces handling, and hence minimizes pipetting steps and contamination risks. Second generation commercial reaction mixes such as those evaluated in this study have now made it far easier for assay set-up, with fewer steps, less time, fewer risks of contamination or error, while improving the reproducibility and reliability of the assays.

One limitation of our study was that we did not evaluate the master mixes utilizing the optimal conditions prescribed by each respective kit manufacturer. As the goal of this study was to identify suitable alternatives, its design defaulted to the conditions currently used with the optimized BSAT assays. Future studies should evaluate commercial kits utilizing the optimized assay conditions, as well as the manufacturers' recommended conditions.

This joint-agency study was conducted to find equivalent or superior alternatives to our current real-time PCR master mix reagents to ensure that we will have sufficient product capacity in the case of a surge event. At completion, our analysis has identified, at a minimum, four additional sources of superior master mix that can be used on the 7500 Fast Dx instrument in the case of a surge event: the TaqMan Fast Universal PCR master mix from Applied Biosystems, the FAST qPCR master mix from Eurogentec, the EXPRESS qPCR SuperMix Universal kit from Invitrogen, and the QuantiFast Probe PCR kit from Qiagen. Of these four kits, the Qiagen kit demonstrated the best cross-platform portability from the 7500 Fast Dx to two additional instruments, the LightCycler and SmartCycler. While all of the kits evaluated in this study have features which laboratories may wish to consider, we found these four kits to be the best alternatives to our current master mix.

\section{Disclaimer statement}

"The findings and conclusions in this report are those of the authors and do not necessarily represent the official position of the Centers for Disease Control and Prevention/the Agency for Toxic Substances and Disease Registry, nor the U.S. Army. Names of vendors or manufacturers are provided as examples of available product sources; inclusion does not imply endorsement of the vendors, manufacturers or products by the Centers for Disease Control and Prevention, the U.S. Department of Health and Human Services, the U.S. Army, or the Department of Defense."

B. anthracis, B. melitensis, B. mallei, and F. tularensis are select agents and their possession, use, and transfer are regulated by the U.S. Department of Health and Human Services, Centers for Disease Control and Prevention, and/or the U.S. Department of Agriculture, Animal and Plant Health Inspection Service. The select agent regulations have mandatory reporting requirements for identification of select agents in diagnostic specimens.

\section{Acknowledgements}

We would like to thank the following USAMRIID researchers for supplying materials and helpful suggestions: Tim Minogue, Gerald Howe, Kitty Baldwin and Deanna Christensen. We thank Harvey Holmes and Judy Sheldon for their contributions to this study. We also thank the following $\mathrm{CDC}$ researchers for their editorial comments: JanettaHakovirta, David Sue, Kenyatta Stephens and Roblena Walker.

\section{References}

[1] C.J. Gerard, K. Olsson, R. Ramanathan, C. Reading, E.G. Hanania, Improved quantitation of minimal residual disease in multiple myeloma using real-time polymerase chain reaction and plasmid-DNA complementarity determining region III standards, Cancer Res. 58 (1998) 3957-3964.

[2] M. Puig, K. Mihalik, M.Y. Yu, S.M. Feinstone, M.E. Major, Sensitivity and reproducibility of HCV quantitation in chimpanzee sera using TaqMan real-time PCR assay, J. Virol. Methods 105 (2002) 253-263.

[3] S.J. Wall, D.R. Edwards, Quantitative reverse transcription-polymerase chain reaction (RT-PCR): a comparison of primer-dropping, competitive, and real-time RT-PCRs, Anal. Biochem. 300 (2002) 269-273.

[4] S.A. Bustin, Quantification of nucleic acids by PCR, in: S.A. Bustin (Ed.), A-Z of Quantitative PCR, International University Line, La Jolla, CA, 2004, pp. 16-17.

[5] Y. Sohni, S. Kanjilal, V. Kapur, Performance evaluation of five commercial realtime PCR reagent systems using TaqMan assays for $B$. anthracis detection, Clin. Biochem. 41 (2008) 640-644.

[6] K.W. Stephens, R.J. Hutchins, L.A. Dauphin, Cross-platform evaluation of commercial real-time reverse transcription PCR master mix kits using a quantitative $5^{\prime}$ nuclease assay for Ebola virus, Mol. Cell. Probes 24 (2010) 370-375.

[7] A.S. Khan, S. Morse, S. Lillibridge, Public-health preparedness for biological terrorism in the USA, Lancet 356 (2000) 1179-1182.

[8] L.D. Rotz, J.M. Hughes, Advances in detecting and responding to threats from bioterrorism and emerging infectious disease, Nat. Med. 10 (2004) S130-S136.

[9] U.S. Department of Health and Human Services, CDC, NIH, Biosafety in Microbiological and Biomedical Laboratories, Washington, DC, 5th ed., 2007.

[10] V.G. DelVecchio, V. Kapatral, P. Elzer, G. Patra, C.V. Mujer, The genome of Brucella melitensis, Vet. Microbiol. 90 (2002) 587-592.

[11] W.C. Nierman, D. DeShazer, H.S. Kim, H. Tettelin, K.E. Nelson, T. Feldblyum, R.L. Ulrich, C.M. Ronning, L.M. Brinkac, S.C. Daugherty, T.D. Davidsen, R.T. Deboy, G. Dimitrov, R.J. Dodson, A.S. Durkin, M.L. Gwinn, D.H. Haft, H. Khouri, J.F. Kolonay, R. Madupu, Y. Mohammoud, W.C. Nelson, D. Radune, C.M. Romero, S. Sarria, J. Selengut, C. Shamblin, S.A. Sullivan, O. White, Y. Yu, N. Zafar, L. Zhou, C.M. Fraser Structural flexibility in the Burkholderia mallei genome, Proc. Natl. Acad. Sci. U.S.A. 101 (2004) 14246-14251.

[12] P. Larsson, P.C. Oyston, P. Chain, M.C. Chu, M. Duffield, H.H. Fuxelius, E. Garcia, G. Halltorp, D. Johansson, K.E. Isherwood, P.D. Karp, E. Larsson, Y. Liu, S. Michell, J. Prior, R. Prior, S. Malfatti, A. Sjostedt, K. Svensson, N. Thompson, L. Vergez, J.K. Wagg, B.W. Wren, L.E. Lindler, S.G. Andersson, M. Forsman, R.W. Titball, The complete genome sequence of Francisella tularensis, the causative agent of tularemia, Nat. Genet. 37 (2005) 153-159.

[13] E. Bode, W. Hurtle, D. Norwood, Real-time PCR assay for a unique chromosomal sequence of Bacillus anthracis, J. Clin. Microbiol. 42 (2004) 5825-5831.

[14] D.R. Christensen, L.J. Hartman, B.M. Loveless, M.S. Frye, M.A. Shipley, D.L. Bridge, M.J. Richards, R.S. Kaplan, J. Garrison, C.D. Baldwin, D.A. Kulesh, D.A. Norwood, Detection of biological threat agents by real-time PCR: comparison of assay performance on the R.A.P.I.D., the LightCycler, and the Smart Cycler platforms, Clin. Chem. 52 (2006) 141-145.

[15] M.P. Ulrich, D.A. Norwood, D.R. Christensen, R.L. Ulrich, Using real-time PCR to specifically detect Burkholderia mallei, J. Med. Microbiol. 55 (2006) 551-559.

[16] W. Abu Al-Soud, P. Radstrom, Capacity of nine thermostable DNA polymerases to mediate DNA amplification in the presence of PCR-inhibiting samples, Appl. Environ. Microbiol. 64 (1998) 3748-3753.

[17] P. Wolffs, H. Grage, O. Hagberg, P. Radstrom, Impact of DNA polymerases and their buffer systems on quantitative real-time PCR, J. Clin. Microbiol. 42 (2004) 408-411.

[18] D. Schoder, A. Schmalwieser, G. Schauberger, M. Kuhn, J. Hoorfar, M. Wagner, Physical characteristics of six new thermocyclers, Clin. Chem. 49 (2003) 960-963.

[19] D. Schoder, A. Schmalwieser, G. Schauberger, J. Hoorfar, M. Kuhn, M. Wagner, Novel approach for assessing performance of PCR cyclers used for diagnostic testing, J. Clin. Microbiol. 43 (2005) 2724-2728.

[20] S. Lu, A.P. Smith, D. Moore, N.M. Lee, Different real-time PCR systems yield different gene expression values, Mol. Cell. Probes 24 (2010) 315-320.

[21] H.A. Bentley, D.R. Belloni, G.J. Tsongalis, Parameters involved in the conversion of real-time PCR assays from the ABI prism 7700 to the Cepheid SmartCycler II, Clin. Biochem. 38 (2005) 183-186. 